\title{
PERSPECTIVES
}

SCIENCE AND SOCIETY

\section{Experimental human challenge infections can accelerate clinical malaria vaccine development}

\section{Robert W. Sauerwein, Meta Roestenberg and Vasee S. Moorthy}

Abstract | Malaria is one of the most frequently occurring infectious diseases worldwide, with almost 1 million deaths and an estimated 243 million clinical cases annually. Several candidate malaria vaccines have reached Phase Ilb clinical trials, but results have often been disappointing. As an alternative to these Phase llb field trials, the efficacy of candidate malaria vaccines can first be assessed through the deliberate exposure of participants to the bites of infectious mosquitoes (sporozoite challenge) or to an inoculum of blood-stage parasites (blood-stage challenge). With an increasing number of malaria vaccine candidates being developed, should human malaria challenge models be more widely used to reduce cost and time investments? This article reviews previous experience with both the sporozoite and blood-stage human malaria challenge models and provides future perspectives for these models in malaria vaccine development.

Half of the world's population (more than 3 billion people) live in malaria-endemic areas, and an estimated 243 million cases of malaria led to nearly 863,000 deaths in 2008 according to the World Health Organization (WHO) World Malaria Report 2009. There are five species of human malaria parasite: Plasmodium falciparum, Plasmodium vivax, Plasmodium ovale, Plasmodium malariae and Plasmodium knowlesi. Recent evidence indicates that $P$. ovale is composed of two subspecies ${ }^{1}$. Most infections are caused by P. falciparum, which is particularly dominant in sub-Saharan Africa. P. vivax is the most widely spread cause of malaria, being responsible for an estimated 80 million to 300 million cases every year, and thus it accounts for a major burden of disease ${ }^{2}$. Plasmodium parasites are highly prevalent in Asia and South America, where individuals can be infected with more than one malaria parasite species simultaneously. Infective foci of $P$. knowlesi have been identified in the past decade in Malaysia, where P. knowlesi is transmitted from simian hosts to humans.
Plasmodia are transmitted by the bites of infected Anopheles mosquitoes. Control strategies are based on the early diagnosis and treatment of uncomplicated infections with artemisinin-based combination therapies, thereby also decreasing transmission ${ }^{3}$, combined with preventive measures aimed at vector (mosquito) control.

Artemisinin-based combination therapies - such as artemether plus lumefantrine, artesunate plus amodiaquine, artesunate plus mefloquine or artesunate plus sulphadoxine-pyrimethamine - have been the WHO-recommended treatment for uncomplicated $P$. falciparum malaria since the development of widespread resistance to chloroquine and sulphadoxine-pyrimethamine. Unfortunately, in P. falciparum, resistance has been observed to all current antimalarial drugs (amodiaquine, chloroquine, mefloquine, quinine and sulphadoxine-pyrimethamine) and, more recently, also to artemisinin derivatives. For uncomplicated $P$. vivax infection, treatment with chloroquine is recommended in those areas without chloroquine resistance. Artemisinin-based combination therapies can be used as an alternative treatment for chloroquine-resistant $P$. vivax. In these cases, artemether plus sulphadoxine-pyrimethamine is not recommended because $P$. vivax can acquire resistance to sulphadoxinepyrimethamine. To fully eradicate $P$. vivax infection, primaquine must be administered to prevent relapses. $P$. ovale and $P$. malariae infections are treated similarly to $P$. vivax infections, although there is no need for primaquine treatment in patients who are infected with $P$. malariae, as this species does not form dormant or latent hypnozoites in hepatocytes (see the WHO Guidelines for the Treatment of Malaria).

Vector control is the primary intervention for decreasing malaria transmission at the community level. When universal vector control coverage is achieved by impregnating bed nets and spraying indoor surfaces of houses with insecticides, malaria transmission can be decreased to close to zero. Unfortunately, the increasing resistance of mosquitoes to insecticides such as dichlorodiphenyltrichloroethane (DDT) and pyrethroids, particularly in Africa, poses challenges to current prevention policies (see the WHO World Malaria Report 2009).

In this context, the development of an effective vaccine could make a significant contribution to the fight against malaria. Ambitious goals in this regard have been set by the Malaria Vaccine Technology Roadmap Process, which aims to achieve a licensed first-generation $P$. falciparum malaria vaccine with more than $50 \%$ protective efficacy against severe disease and death, lasting for at least 1 year, by the year 2015. Malaria vaccine development has been fuelled by new technology enabling the sequencing of the $P$. falciparum, $P$. vivax and Anopheles gambiae genomes and the development of experimentally relevant animal models, combined with significant increases in financial resources from funders such as the Bill \& Melinda Gates Foundation, the European Union, the US National Institutes of Allergy and Infectious Diseases and the US Agency for International Development. Currently, there are 38 P. falciparum and two $P$. vivax candidate malaria vaccines or 


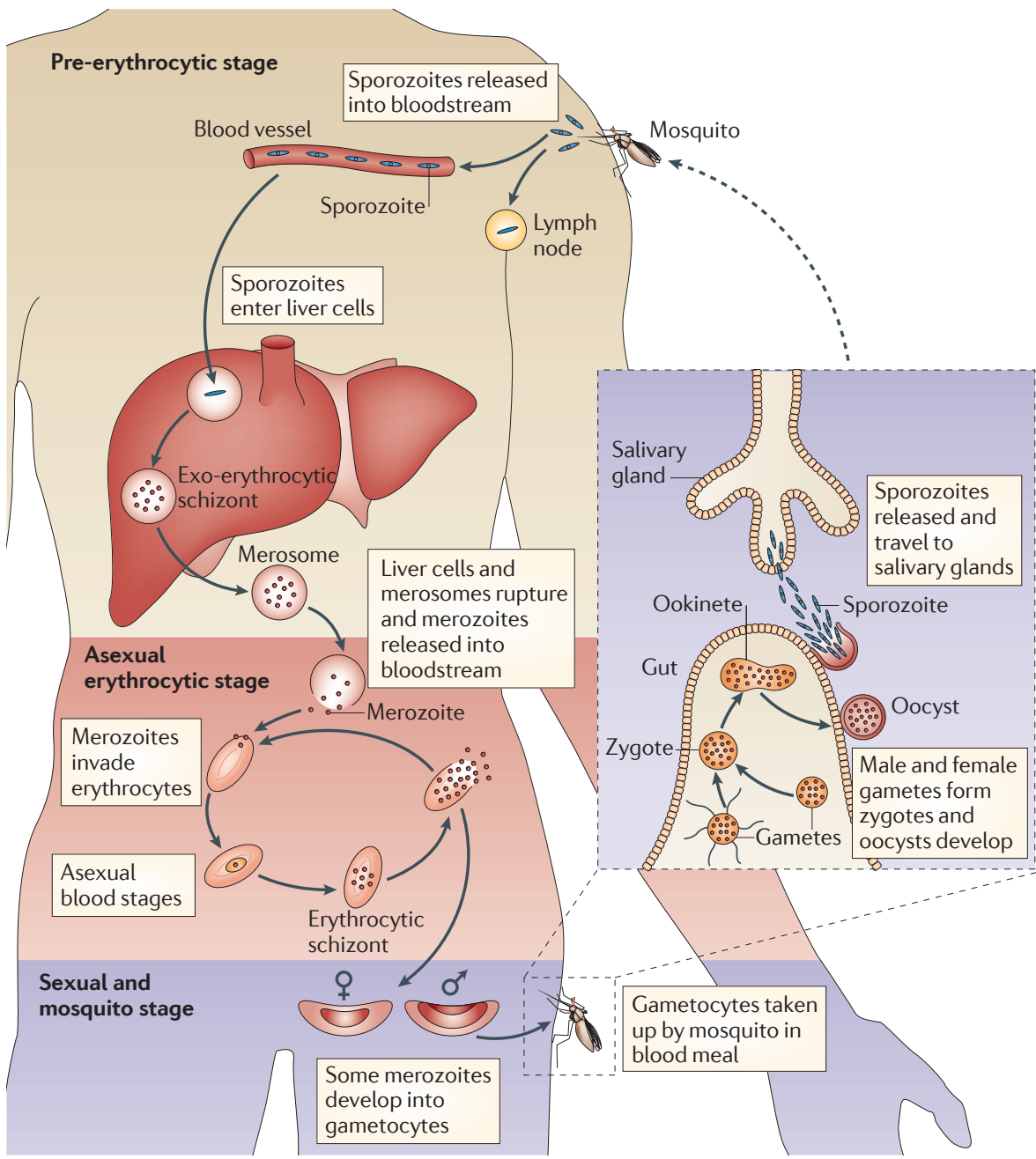

Figure 1| Plasmodium falciparum life cycle showing the three developmental stages of the parasite that are targeted by malaria vaccine candidates. Parasites (sporozoite stage) are injected into the skin by a female Anopheles spp. mosquito. From the skin, a proportion of sporozoites will travel through the bloodstream to the liver. Some sporozoites will be trapped in regional lymph nodes. In hepatocytes, parasites develop and multiply for $6-7$ days before merosomes are budded from the cell and enter the hepatic sinusoids. Merosomes eventually rupture, releasing merozoite-stage parasites that invade erythrocytes for further reproduction. Clinical malaria is caused by the 48-hour cyclical proliferation of asexual-stage parasites in erythrocytes. Malaria mortality is primarily due to organ dysfunction, in particular of the brain, following sequestration of infected erythrocytes in the microvasculature. The development of sexual forms of the parasite (gametocytes) in the blood allows the transmission of parasites to mosquitoes with subsequent bites. Once ingested, the parasite gametocytes taken up in the blood further differentiate into male or female gametes and then fuse in the mosquito gut. This produces an ookinete that penetrates the gut lining and produces an oocyst in the gut wall. When the oocyst ruptures, it releases sporozoites that migrate through the mosquito's body to the salivary glands, where they are then ready to infect a new human host. Image is modified, with permission, from REF. 59 @ (2004) Macmillan Publishers Ltd. All rights reserved.

vaccine components in advanced preclinical or clinical development as listed by the WHO Malaria Vaccine Rainbow Tables.

Malaria vaccine candidates are categorized according to the Plasmodium life cycle stage at which the targeted antigen is expressed (FIG. 1). Pre-erythrocytic stage vaccines aim to prevent the passage of parasites through the human liver and subsequent blood-stage infection, leading to the induction of sterile immunity. Asexual erythrocytic stage vaccines focus on delaying or decreasing parasite multiplication in red blood cells, thereby decreasing morbidity and preventing mortality. Transmissionblocking vaccines consist of sexual- or mosquito-stage antigens that prevent infection passing from humans to mosquitoes, thereby decreasing the spread of malaria in the population.
Generally, less than $10 \%$ of preclinical vaccine projects progress to Phase III clinical evaluation ${ }^{4}$. Clinical development is time consuming and costly. Candidate malaria vaccines are selected downstream of clinical testing on the basis of safety, immunogenicity and, eventually, efficacy profiles. Whereas the first two criteria can generally be assessed in a small initial Phase I trial, field vaccine efficacy can only be assessed in Phase II trials, which require larger study groups in malaria-endemic areas. The sample size of Phase II trials depends on the prevalence of malaria infections in that area and the expected efficacy of the candidate vaccine. Ideally, immunological assays carried out in initial clinical trials should predict potential efficacy in subsequent trials, by analogy with, for example, hepatitis B virus surface antigen (HBsAg)specific antibody titres for the hepatitis $B$ vaccine. However, with the current lack of unequivocal correlates of immune protection against malaria in either animal models or in vitro assays on human samples, there is a continuous need to test field efficacy in time-consuming and costly Phase II trials in malaria-endemic areas. There are only a limited number of competent field trial sites for malaria that can adhere to the good clinical practice guidelines established by the International Conference on Harmonisation of Technical Requirements for Registration of Pharmaceuticals for Human Use (ICH), which describe the monitoring, reporting and archiving responsibilities of all participants in the conduct of clinical trials (see The Malaria Product Pipeline: Planning for the Future). Finally, there seems to be a downward trend in malaria incidence in several endemic areas, most probably as a result of improved policy and adherence to malaria control measures, and this will further increase the size and costs of Phase II field trials ${ }^{5}$.

Human experimental sporozoite infections carried out under strictly controlled laboratory and clinical conditions, in which volunteers are exposed to the bites of laboratory-reared Plasmodium-infected mosquitoes, are an intermediate step between Phase I and Phase II trials, providing information on preliminary vaccine efficacy. It is common practice to test the efficacy of pre-erythrocytic stage malaria vaccine candidates by experimental sporozoite infection before going into the field. In such cases, a distinction is thus made between Phase IIa trials using experimental infection of volunteers in non-endemic areas and Phase IIb field trials in endemic areas. 
Poor preliminary efficacy in Phase IIa trials may subsequently halt progression of the vaccine candidate to Phase IIb trials. By contrast, the efficacy of asexual erythrocytic stage vaccine candidates is generally assessed in field trials only, although blood-stage challenge models have been used.

Here, we present the history of artificial malaria challenge infections, the clinical aspects of $P$. falciparum challenges using sporozoites or blood-stage parasites and experience with $P$. vivax challenges. We discuss the strengths and limitations of both models and provide future perspectives.

\section{Historical perspective}

Deliberate infection of humans with malaria was first carried out in 1917 by Wagner von Jauregg ${ }^{6}$ as a therapy primarily for patients with neurosyphilis, and he was awarded the Nobel Prize in Medicine for his work in 1927. Thousands of patients have undergone this treatment, which was administered by the bites of infectious mosquitoes or by intravenous or subcutaneous inoculation of dissected Plasmodium sporozoites suspended in media. Historically, P. vivax was used most frequently, but infections were also carried out with $P$. falciparum, P. malariae and P. ovale. The objective was to induce a febrile illness that was thought to be beneficial for the prognosis of the disease. This practice stopped with the advent of antibiotics for the treatment of the Treponema pallidum infection that causes syphilis.

In the 1960s, experimental human malaria infections were used to assess the effects of anti-malaria treatments on healthy non-immune male inmates in the United States ${ }^{7}$. Following the discovery of protocols for the continuous culture of P. falciparum in 1976 (REF. 8) and protocols for the generation of mature P. falciparum gametocytes in vitro in 1981 (REF. 9), laboratory-reared infectious mosquitoes could be produced ${ }^{10}$ and human malaria sporozoite infections could be carried out more routinely.

The first well-documented study of human experimental malaria infection with these laboratory-reared infectious mosquitoes was carried out in 1986 at the US Walter Reed Army Institute of Research (WRAIR), the US Naval Medical Research Institute (NMRI) and the US National Institutes of Health (NIH). Six volunteers were infected with $P$. falciparum sporozoites by the bites of infectious Anopheles freeborni and Anopheles stephensi mosquitoes ${ }^{11}$. The following year, the efficacies of the first recombinant protein and synthetic peptide
P. falciparum vaccines were tested in experimentally infected volunteers ${ }^{12,13}$. Since the late 1980s, the number of institutions carrying out experimental infections with P. falciparum sporozoites has been growing. In 2007, data were published from a total of 532 volunteers ${ }^{14}$. So far, unpublished analysis shows that a total of 1,343 volunteers have been experimentally infected with $P$. falciparum between 1985 and 2009 (REF. 15); 526 of these volunteers took part in vaccine trials (TABLE 1), and of these, 118 volunteers were protected against infection by the vaccine candidate. The most successful immunogens were RTS,S (a pre-erythrocytic stage vaccine consisting of the $P$. falciparum circumsporozoite protein combined with HBsAg; developed by GlaxoSmithKline in partnership with PATH Malaria Vaccine Initiative) and irradiated whole parasites delivered by mosquito bite.

\section{Comparison with field trials}

Differences between natural and experimental infections mean that it is important to validate the results of Phase IIa challenge trials with data from Phase IIb field trials in malaria-endemic areas. Only three candidate vaccines have been assessed by both types of trial, allowing a comparison of the protective outcomes. The best studied candidate vaccine, RTS,S, which is currently in Phase III trials, has repeatedly demonstrated a protective efficacy of $\sim 30-50 \%$ in Phase IIa trials with sterile protection as the study end point ${ }^{16-18}$. Interestingly, a similar $\sim 30-50 \%$ efficacy of RTS,S was found in Phase IIb trials in the field using time to first clinical malaria episode as the primary study end point ${ }^{19}$. A similar association between the results of Phase IIa and Phase IIb trials was found when testing long-term protection in adults ${ }^{19,20}$. A second pre-erythrocytic stage candidate vaccine, ME-TRAP (a multi-epitope string fused to thrombospondin-related adhesion protein), delivered by a DNA prime and attenuated poxvirus boost, induced complete protection in only a few volunteers (three out of 74) in Phase IIa trials, and no protection was found in adult Phase IIb field studies in the Gambia ${ }^{21,22}$. Artificial blood-stage challenge has been used in a Phase II trial after immunization with Combination B (a combination of merozoite surface protein 1 (MSP1), MSP2 and ring-infected erythrocyte surface antigen (RESA)) in 17 volunteers, which resulted in no decrease in parasite growth rates ${ }^{23,24}$; this is in line with results from a Phase IIb trial of Combination B conducted in Papua New
Guinea $^{25}$. These limited data indicate that results obtained in experimental challenges are generally in line with results in the field, but more comparisons are required before definite conclusions can be drawn.

\section{Experimental sporozoite infection}

The delivery of sporozoite-stage malaria parasites by mosquito bites has traditionally been used as a model to test pre-erythrocytic stage vaccines. Since the late 1980s, standardization of experimental sporozoite infections has improved and efforts to further increase harmonization are ongoing. Such infections are currently routinely carried out at: the US Military Malaria Vaccine Program; the University of Maryland, USA; Radboud University Nijmegen Medical Centre (RUNMC), the Netherlands; the University of Oxford, UK; and, more recently, Seattle Biomed, USA ${ }^{15}$. All centres use A. stephensi mosquitoes that feed on either the chloroquine-sensitive NF54 strain of $P$. falciparum or the 3D7 clone of NF54. In addition, limited numbers of volunteers have been challenged with the 7G8 strain of P. falciparum ${ }^{14}$.

Approximately 14-21 days after feeding, mosquitoes are checked for infection by microscopic examination of salivary glands. Healthy human volunteers are subsequently exposed to the bites of five infectious mosquitoes for either 5 or 10 minutes (FIG. 2). Almost $100 \%$ of volunteers bitten by five infected mosquitoes develop patent parasitaemia, with very rare exceptions ${ }^{26,27}$. Infection rates drop significantly when volunteers are exposed to fewer than five infected mosquitoes ${ }^{27,28}$.

After infection, subjects are monitored closely on an outpatient basis. Signs and symptoms such as headache, myalgia and fever are noted, and a physical examination and thick blood smears (a drop of blood on a glass slide) are carried out once, twice or thrice daily. The period before blood-stage parasites can be detected in thick smears by microscopy (the prepatent period) ranges from 7 to 20 days, with a mean of approximately 11 days ${ }^{7,14,26}$. As soon as parasites are microscopically detected, volunteers are treated with a curative regimen of chloroquine, artemether plus lumefantrine, or atovaquone plus proguanil. Nearly all volunteers will develop symptoms of clinical malaria infection; approximately one-fifth of volunteers temporarily develop symptoms graded as severe (symptoms that prevent daily activities), but severe or life-threatening malaria has never occurred ${ }^{26}$. The most common symptoms are fatigue and headache, 


\begin{tabular}{|c|c|c|c|c|c|c|c|}
\hline Vaccine & $\begin{array}{l}\text { Plasmodium } \\
\text { protein }\end{array}$ & Category & $\begin{array}{l}\text { Number of } \\
\text { volunteers } \\
\text { challenged }\end{array}$ & $\begin{array}{l}\text { Number of } \\
\text { volunteers } \\
\text { protected }\end{array}$ & $\begin{array}{l}\text { Year of } \\
\text { publication }\end{array}$ & Institution or company & Refs \\
\hline Several products & CSP & Pre-erythrocytic & 317 & $94(29.65 \%)$ & 1987-2009 & $\begin{array}{l}\text { University of Maryland; WRAIR*; } \\
\text { University of Oxford, UK; Johns } \\
\text { Hopkins University School of } \\
\text { Hygiene and Public Health, } \\
\text { Maryland, USA; NMRI*; University } \\
\text { of Lausanne, Switzerland }\end{array}$ & $\begin{array}{r}12,13,16, \\
18,20,22, \\
40,45,62, \\
66-73\end{array}$ \\
\hline Several products & TRAP & Pre-erythrocytic & 74 & $3(4.05 \%)$ & 2003-2006 & University of Oxford & $22,74,75$ \\
\hline $\begin{array}{l}\text { AMA1 with } \\
\text { AS02A or AS01B }\end{array}$ & AMA1 & $\begin{array}{l}\text { Asexual } \\
\text { erythrocytic }\end{array}$ & 16 & $0(0 \%)$ & 2009 & $\begin{array}{l}\text { US Military Malaria Vaccine } \\
\text { Program }\end{array}$ & 35 \\
\hline $\begin{array}{l}\text { FFM ME-TRAP } \\
\text { plus PEV3A }\end{array}$ & $\begin{array}{l}\text { CSP, TRAP and } \\
\text { AMA1 }\end{array}$ & $\begin{array}{l}\text { Pre-erythrocytic } \\
\text { and asexual } \\
\text { erythrocytic }\end{array}$ & 24 & $0(0 \%)$ & 2008 & University of Oxford & 78 \\
\hline $\begin{array}{l}\text { SPf(66)30 or } \\
\text { SPf(105)20 with } \\
\text { Alum }\end{array}$ & MSP & $\begin{array}{l}\text { Asexual } \\
\text { erythrocytic }\end{array}$ & 9 & $0(0 \%)^{\ddagger}$ & 1988 & $\begin{array}{l}\text { Universidad Nacional de } \\
\text { Colombia }\end{array}$ & 79 \\
\hline MuStDO 5 & $\begin{array}{l}\text { CSP, EXP1, SSP2, } \\
\text { LSA1, LSA3 }\end{array}$ & Pre-erythrocytic & 31 & $0(0 \%)$ & 2005 & Naval Medical Research Center* & 80 \\
\hline $\begin{array}{l}\text { FMP1 with } \\
\text { AS02A }\end{array}$ & MSP1 & $\begin{array}{l}\text { Asexual } \\
\text { erythrocytic }\end{array}$ & Unknown & $0(0 \%)$ & 2005 & WRAIR* & 81 \\
\hline
\end{tabular}

Alum, aluminium hydroxide adjuvant (Alhydrogel; Brenntag biosector); AMA1, apical membrane antigen 1; AS01, GlaxoSmithKline adjuvant system 01; CSP, circumsporozoite protein; EXP1, exported protein 1; FFM ME-TRAP, multi-epitope string fused to TRAP that is delivered in fowlpox virus strain FP9 and modified vaccinia virus Ankara vectors in prime-boost combinations; FMP1, carboxy-terminal region of MSP1; LSA, liver-stage antigen; LSA1-NRC, full-length carboxy- and amino-terminal flanking domains and two of the 17 amino acid repeats from the central repeat region of LSA1; MSP, merozoite surface protein; MuStDO 5, multi-stage DNA vaccine operation 5 antigens; NMRI, Naval Medical Research Institute, USA; NYVAC-Pf7, a highly attenuated vaccinia virus with seven P. falciparum genes inserted into its genome; PEV3A, virosomal formulation of CSP and AMA1; Pfs25, 25kDa ookinete surface antigen; SERA, serine-repeat antigen protein; SSP2, sporozoite surface protein 2; SPf, synthetic P. falciparum peptides of MSP; TRAP, thrombospondin-related adhesion protein; WRAIR, Walter Reed Army Institute of Research, USA. ${ }^{\star}$ Currently the US Military Malaria Vaccine Program. ${ }^{*}$ Three of five volunteers immunized with SPf(66)30 eventually cleared parasitaemia after they experienced asexual parasitaemia that was detectable by microscopy.

and severe symptoms can include headache, fatigue, malaise, chills, myalgia, rigors, nausea and vomiting. Clinical symptoms generally coincide with the detection of blood-stage parasites at densities of 10-20 parasites per $\mu$ l of blood by microscopy of thick blood smears ${ }^{26}$. This corresponds to a parasitaemia of approximately $0.0004 \%$. Severe malaria is generally diagnosed when parasitaemia is 3 to 4 logs greater than the peak parasitaemia in challenge trials. After the start of malaria treatment, symptoms can temporarily increase in severity but subside quickly with an average duration of approximately 2-3 days.

Routine laboratory checks generally show a moderate decrease in leukocyte and platelet numbers during infection, with no change in haemoglobin concentration ${ }^{27}$. Bleeding or thrombogenic complications have never been described ${ }^{26,27}$.
Abnormalities of liver enzymes have been observed, but these abnormalities did not result in clinical manifestations and they resolved after a few days ${ }^{26,27}$.

Immediate treatment of volunteers at the earliest phase of microscopically detectable blood-stage infection ensures that the potential risks of complications associated with severe malaria are minimized to the greatest extent possible. Indeed, human malaria challenge infections have been shown to be safe in the 1,343 volunteers challenged so far ${ }^{14,26,27}$. Recently, safety concerns were raised because of a cardiac event in a young volunteer shortly after treatment for diagnosed malaria, although a definite relationship between the cardiac event and the experimental malaria infection was not established ${ }^{29}$. Nevertheless, it has been generally agreed that volunteers with an increased risk of cardiac disease should be excluded from such trials.
In addition to the clinical manifestations, participation in an experimental sporozoite infection trial has a major impact on the daily life of volunteers, particularly because of the intense follow-up with blood sampling several times daily. Volunteers' perception of their participation in such a trial depends mainly on whether they have realistic expectations of trial procedures and the severity of symptoms, indicating the importance of providing accurate and sufficient information to volunteers before the onset of the trial.

\section{Measurement of parasitaemia}

A real-time quantitative PCR assay based on $18 \mathrm{~S}$ ribosomal RNA gene transcripts has been developed for tracking the kinetics of developing parasitaemia before a positive diagnosis of infection can be made from a thick blood smear using microscopy ${ }^{30}$. This 
assay is becoming increasingly important for assessing very low parasite densities and incremental changes in density in smallscale Phase IIa trials ${ }^{31}$. The detection of parasites below microscopy thresholds by PCR allows for a detailed analysis of cyclical parasite growth in the blood, albeit for a short time window of 2-3 days between liver-stage infection and microscopic detection $^{30}$. Several statistical models have been developed to further analyse profiles of parasitaemia and partial protection in vaccine trials ${ }^{32-34}$. For example, these models allow a separate estimation of liver-stage and blood-stage parasite development. From the first wave of parasite DNA that is detected in the blood, an estimation can be made of the number of merozoites released from the liver, making it possible to approximate the extent of pre-erythrocytic stage inhibition resulting from a vaccine (simulated in FIG. 3a). Similarly, the ratio of parasite DNA between the second and first replication cycles in the blood reflects the growth rate of blood-stage parasites. Comparing these ratios between test subjects and controls can indicate inhibitory effects of a vaccine candidate on the growth of blood-stage parasites (simulated in FIG. 3b). Such analyses could be of particular interest in trials of multi-stage vaccines (combining both liver- and blood-stage antigens) to assess stage-specific protective immunity. For example, statistical modelling of parasitaemia from a recent Phase IIa trial with the vaccine candidate apical membrane antigen 1 (AMA1), a protein that is mainly expressed by blood-stage parasites, indicates inhibition of pre-erythrocytic parasite stages $^{35}$, which highlights the possible role of sporozoite-expressed AMA1 in disease progression $^{36}$.

\section{Experimental blood-stage infection}

The evaluation of asexual erythrocytic stage vaccine candidates requires follow-up of blood-stage parasitaemia over a sufficiently lengthy period of time to determine parasite growth rates. This requirement could compromise the safety of volunteers, as blood-stage parasitaemia is responsible for malaria morbidity and even mortality. In currently accepted protocols, the appearance of Plasmodium-infected erythrocytes in thick blood smears examined microscopically leads to immediate initiation of treatment with curative anti-malarial drugs. Harbouring higher numbers of parasites in the bloodstream increases the risks to volunteer safety, so the length of the observation period for parasite multiplication in erythrocytes is limited.

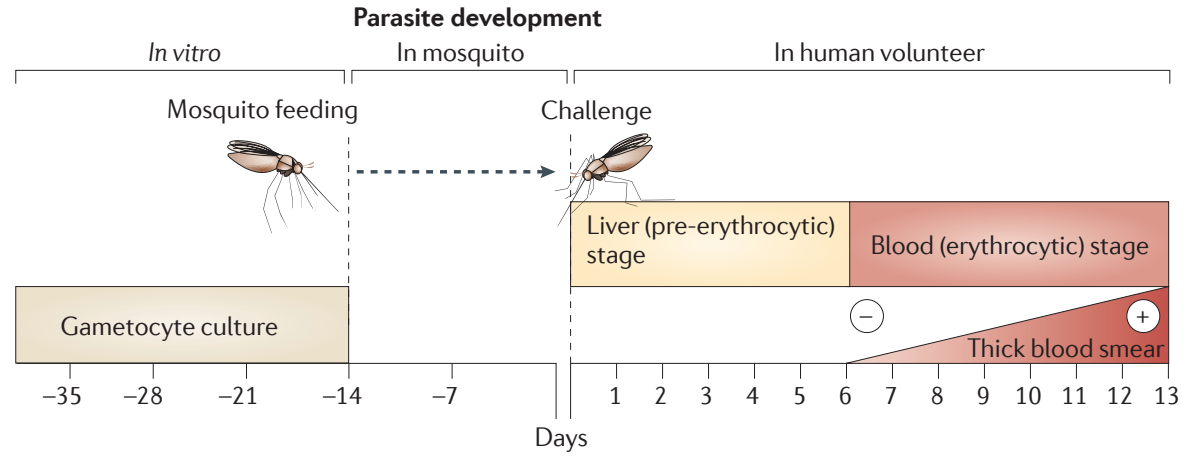

Figure 2 | Timeline of Plasmodium falciparum sporozoite challenge infection in humans. Gametocytes are derived from in vitro parasite culture in donor blood and are fed to laboratory-reared Anopheles stephensi mosquitoes. After 14-21 days, five infectious mosquitoes are allowed to feed on malaria-naive human volunteers for 5-10 minutes. Subsequent development of liver-stage parasites is subclinical and takes approximately 6 days. Parasites can be detected in the blood of unprotected volunteers by microscopy (using a thick blood smear) on average 11 days (range 7-15 days) after challenge.

A possible solution is the intravenous inoculation of very small numbers of infected erythrocytes, based on the idea that such a low level of sub-microscopic parasitaemia will not result in clinical risks and will allow extended follow-up of parasite replication in erythrocytes. The number of parasites that are inoculated is approximately 1,000 times lower than the estimated number of merozoites released from the liver following a standard experimental sporozoite challenge with bites from five infected mosquitoes. This allows for an extended blood-stage follow-up of approximately three more replication cycles (6 days) before thick blood smear detection thresholds are reached, with obligatory treatment.

A master cell bank of infected erythrocytes for human clinical use has been generated by storing infected erythrocytes from two parasitaemic volunteers who were infected by mosquito bites, in compliance with blood bank safety criteria ${ }^{37}$. Since the 1990s, approximately 50 humans have been infected by direct inoculation of infected erythrocytes from this master cell bank. The length of the prepatent period - the interval from inoculation until infected erythrocytes are microscopically detectable - correlates with the number of inoculated parasites ${ }^{7}$. The sensitivity of the model has been further increased by the administration of very small inoculae of infected erythrocytes, combined with the introduction of the quantitative real-time PCR assay for measuring parasite growth rates during this sub-microscopic period ${ }^{37,38}$. With inoculae as small as 300 infected erythrocytes, parasite growth curves were generated over a 7-9-day period before initiation of treatment was required ${ }^{37}$.
The blood-stage challenge model has several potential shortcomings. The viability of the injected parasites can only be determined retrospectively by culture, so it is difficult to standardize the exact number of viable injected parasites. Differences of a factor of ten in terms of the number of viable parasites have been described between inoculae $\mathrm{e}^{38,39}$. Furthermore, although the small number of inoculated parasites allows for a long window of observation, it may also boost the immune response, and low-level blood-stage infections are very efficient at inducing complete protection ${ }^{40}$. Finally, the liver stage of parasite development is circumvented by this model, bypassing potential immune effects induced by the vaccine on liverstage parasites. This may be important, as some asexual erythrocytic stage vaccine candidate antigens can also be expressed during the liver stage ${ }^{36}$. However, irrespective of these disadvantages, low-dose blood-stage challenges allow sufficient time to monitor several parasite multiplication cycles. With further validation, they might function as a crucial decision point for progress to Phase IIb trials, thereby saving time and money, and decreasing the requirement for Phase IIb trial subjects. So far, only one asexual erythrocytic stage vaccine has been tested by blood-stage challenge $e^{24}$. The results of a second trial with the vaccine AMA1 carried out at the University of Oxford, UK, will soon be reported (ClinicalTrials.gov identifier: NCT00984763). A direct comparison between blood-stage and sporozoite-stage challenge infections will be helpful to determine the most suitable model to test such asexual erythrocytic stage vaccines. 


\section{a Pre-erythrocytic stage vaccine}

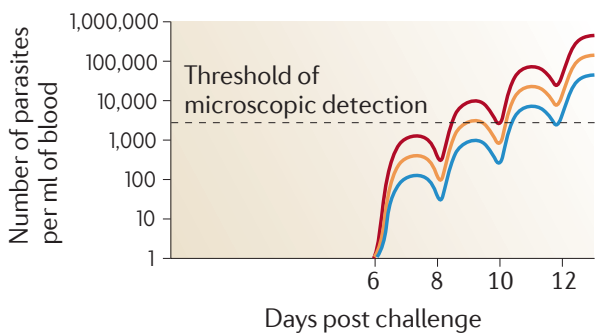

Days post challenge b Asexual erythrocytic stage vaccine

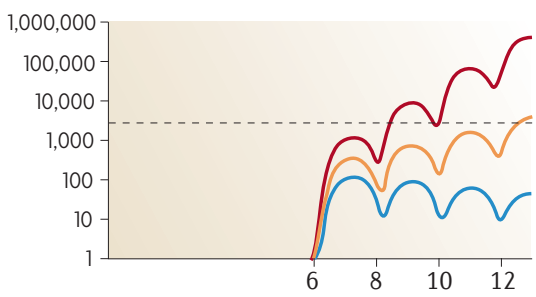

Days post challenge

$-0 \%$ inhibition $-70 \%$ inhibition $\quad-90 \%$ inhibition

Figure 3 | Simulated effects of immunization on parasite growth in the peripheral blood of volunteers after Plasmodium falciparum sporozoite challenge, based on statistical modelling. The kinetics of parasite growth after immunization depend on which parasite stage the vaccine targets. We simulated the effect of a pre-erythrocytic (a) or asexual erythrocytic (b) stage vaccine on cyclical parasite growth in peripheral blood. The effects of $0 \%, 70 \%$ or $90 \%$ inhibition on parasite numbers are shown. After sporozoite challenge, the kinetics of blood-stage parasitaemia can be evaluated by quantitative real-time PCR up to the threshold of detection of parasites in the blood by microscopy (thick blood smear); this threshold is approximately $4 \times 10^{3}$ parasites per $\mathrm{ml}$ of blood. A careful comparison of blood-stage parasitaemia between immunized and control volunteers in a sporozoite challenge trial will allow investigators to distinguish between pre-erythrocytic or asexual erythrocytic stage inhibition. Furthermore, the percentage inhibition can be estimated. Image is modified, with permission, from REF. 32 ๑) (2004) The American Society of Tropical Medicine and Hygiene.

\section{Plasmodium vivax infection}

Although the first experimental human malaria infections were carried out with $P$. vivax ${ }^{41}$, the standardization of $P$. vivax challenge for routine use has proven to be much more difficult than for $P$. falciparum challenge. A major hurdle is the absolute requirement for reticulocytes or young erythrocytes to obtain long-term in vitro growth of $P$. vivax.

Nevertheless, promising results have been obtained through an alternative approach in which experimental infections are initiated using wild-type $P$. vivax parasites obtained from infected humans in Colombia. Blood from P. vivax-infected patients was assessed using routine blood bank procedures to exclude the presence of other transmissible agents (such as T. pallidum, hepatitis B virus and hepatitis $C$ virus) and was subsequently fed to laboratory-reared Anopheles albimanus mosquitoes. After 14-15 days, mosquitoes were allowed to feed for 10-15 minutes on the forearms of healthy human volunteers. A total of 40 non-immune volunteers took part in two different trials, and data from 17 volunteers have been published so far ${ }^{15,42}$. After microscopic detection of parasites by thick blood smear, all participants were treated with a combination of chloroquine and primaquine. Because a proportion of $P$. vivax parasites can lay dormant as hypnozoites in the liver or develop slowly in humans, resulting in long prepatent periods ${ }^{23}$, primaquine is prescribed to ensure clearance of all liver-stage parasites. This complicated protocol may be further compromised by drug resistance of some $P$. vivax strains, as is commonly observed in Southeast Asia.

The most frequently reported symptoms were myalgia, headache and malaise, without the occurrence of severe or serious adverse events. The prepatent period was $9-13$ days $^{42}$. The $P$. vivax challenge model has been further developed in the US Military Malaria Vaccine Program by the transportation of freshly infected Anopheles dirus mosquitoes from the ThaiBurmese border to infect malaria-naive volunteers in the United States (ClinicalTrials. gov identifier: NCT00935623). Currently, the first $P$. vivax vaccine candidate, based on the $P$. vivax circumsporozoite protein VMP001, is being tested by such challenge studies in the United States (ClinicalTrials. gov identifier: NCT01157897). Quantitative real-time PCR detection of $P$. vivax parasite load and genotyping of wild-type parasites will further improve the $P$. vivax challenge mode ${ }^{15}$. Hopefully, the future development of new laboratory tools, including the use of stem cells as a source for young erythrocytes, will facilitate the long-term in vitro culture of $P$. vivax.

\section{Strengths and limitations}

Experimental human challenges aim to predict the potential efficacy of vaccine candidates against natural infections in the field. A major strength of the sporozoite infection model is the use of infectious mosquitoes, mimicking the natural route of infection. Moreover, human experimental challenges are carried out in a controlled environment, allowing detailed evaluation of parasite growth and immunological determinants. In addition to the evaluation of vaccine efficacy, experimental challenges provide the opportunity to study correlates and mechanisms of protection. An example is the induction of immunity using whole parasites, by exposure of malaria-naive volunteers to infectious mosquito bites while using chloroquine prophylaxis ${ }^{43}$. Chloroquine kills blood-stage parasites but leaves liver-stage parasites unaffected, thereby exposing the liver-stage and early blood-stage antigens to the immune system. Subsequent challenge showed that volunteers were completely protected from infection, and this was associated with multi-functional memory $\mathrm{T}$ cell responses. However, such immunization protocols are not practical for use in the field because parasite inoculation cannot be controlled and chloroquine resistance is widespread.

Several differences between experimental and naturally acquired infections might limit the interpretation of results from experimental challenge models. First, experimental infections are carried out using one parasite strain only, whereas it is well accepted that $P$. falciparum field strains are genetically diverse within and between regions ${ }^{44}$. Genetic diversity of the parasite strains is a major challenge for protein-based vaccines that target strain-specific antigens, and puts limitations on the direct translation of results from Phase IIa trials into the field situation. The availability of a small portfolio of genetically well-characterized $P$. falciparum strains for experimental infections would be a major asset. Trials to test such strains in humans are currently being carried out. Another potential difference is that in an experimental infection the parasite load is delivered almost instantly by five infected mosquitoes. Such a high parasite burden has been considered unnatural and might be an overly stringent test for the protective capacity of the vaccine-induced immune response ${ }^{45}$. However, although the frequency of infectious mosquito bites is generally less than this in malaria-endemic areas, intense transmission can occur. A person may be subjected to 35-96 mosquito bites per night, and in certain areas approximately $10 \%$ of mosquitoes are infected with P. falciparum ${ }^{46}$. 
A final potential limitation of current malaria challenge models involving sporozoite infection relates to the uncontrolled number of sporozoites inoculated by biting mosquitoes. This number is generally thought to vary up to a maximum of several thousand sporozoites $^{47-51}$. Use of a well-defined number of inoculated sporozoites will strengthen the power of the model, as the dose probably influences the prepatent period ${ }^{7,27,52}$.

In principle, the most accurate way of dosing sporozoites is to inject them directly by needle and syringe, as the number of sporozoites counted in mosquito salivary glands or the number of mosquito bites is a poor predictor of the number of sporozoites injected ${ }^{49}$. Early in the practice of malaria therapy, sporozoites were extracted from mosquito salivary glands (in 1927) ${ }^{53}$ and the number of injected sporozoites was determined (in 1937) ${ }^{54}$. However, standardized sporozoite viability assays are not yet available. Recent progress has been made by Sanaria Inc..$^{55}$, which has developed technology for the purification and cryopreservation of aseptic sporozoites for use in humans according to the current safety standards. The first results of a human challenge study with aseptic P. falciparum sporozoite-infected mosquitoes indicate that these mosquitoes might be very efficient at conveying infection ${ }^{56}$. Experimental human infections are underway to test the infectiousness of these cryopreserved sporozoites by needle injection. However, one must bear in mind that needle and syringe administration of a bolus of sporozoites is clearly different from mosquito bite delivery, which may be an important factor to consider particularly for sporozoite vaccines that aim to induce antibodies to immobilize sporozoites.

\section{Conclusions and perspectives}

Experimental human infections provide a crude model to predict malaria vaccine efficacy in future field trials in a well-controlled setting. The experimental malaria challenge model in humans using $P$. falciparuminfected mosquito bites is now well established in several international sites and increasingly used as a crucial check point for the clinical development of pre-erythrocytic stage malaria vaccines. Taking into account the potential limitations, such efficacy data from Phase IIa trials will support the decisionmaking process by ethical boards and communities in malaria-endemic countries regarding whether to further test a candidate vaccine in Phase IIb trials in susceptible populations. In addition to vaccine safety data, the availability of information on potential efficacy is an important asset for ethical justification to conduct experimental malaria infections in human volunteers. In vaccine research, most risk is borne by study subjects and the benefits accrue mainly to the community in finding safe and protective vac$\operatorname{cines}^{57}$. The only candidate malaria vaccine showing protective efficacy in Phase IIb field trials so far is RTS,S. This candidate vaccine would almost certainly never have been developed without optimization in a series of Phase IIa trials. As is true for any type of clinical research, risks must be minimized and scientific benefits maximized. We believe that the benefits of Phase IIa trials outweigh the potential risks in well-designed studies and will be essential to the development of an effective malaria vaccine, provided that all safeguards are in place for the safety of volunteers ${ }^{58}$.

The more recent introduction of a sensitive PCR assay for parasite detection has enhanced the reproducibility and statistical power of human challenge infections. Statistical models will be applied to further improve the discriminative power between control and test groups as well as to provide biological information about the parasite life cycle (including the duration of liver-stage maturation, number of infected hepatocytes, duration of blood-stage trophozoite maturation and multiplication rates). Initiatives are underway to further strengthen and harmonize the human challenge model, with possible applications for testing asexual erythrocytic stage vaccines and for $P$. vivax vaccine research ${ }^{15}$. As there is substantial variation in the numbers of sporozoites that are injected by mosquitoes and this cannot be controlled in the sporozoite challenge model, administration of a known number of sporozoites by needle injection may be a further improvement to the model. In addition, the human challenge model will benefit from the availability of a small portfolio of genetically well-characterized strains to explore immune responses to different strains and heterologous protection.

Such advances will accelerate malaria vaccine development, with the aim of meeting the ambitious goals of the Malaria Vaccine Technology Roadmap by 2015-2025.

Robert W. Sauerwein and Meta Roestenberg are at the Department of Medical Microbiology, Radboud University Nijmegen Medical Centre, P.O. BOX 9101, 6500 HB Nijmegen, The Netherlands.

Vasee S. Moorthy is at the Initiative for Vaccine Research, World Health Organization, 20 Avenue Appia, 1211 Geneva 27, Switzerland.

Correspondence to R.W.S. e-mail: R.Sauerwein@mmb.umcn.nI doi: $10.1038 /$ nri2902
Sutherland, C. J. et al. Two nonrecombining sympatric forms of the human malaria parasite Plasmodium ovale occur globally. J. Infect. Dis. 201, 1544-1550 (2010).

2. Mueller, l. et al. Key gaps in the knowledge of Plasmodium vivax, a neglected human malaria parasite. Lancet Infect. Dis. 9, 555-566 (2009). 3. Bousema, T. et al. Revisiting the circulation time of Plasmodium falciparum gametocytes: molecular detection methods to estimate the duration of gametocyte carriage and the effect of gametocytocidal drugs. Malar. J. 9, 136 (2010).

4. Davis, M. M. et al. The expanding vaccine development pipeline, 1995-2008. Vaccine 28, 1353-1356 (2010).

5. O'Meara, W. P., Mangeni, J. N., Steketee, R. \& Greenwood, B. Changes in the burden of malaria in sub-Saharan Africa. Lancet Infect. Dis. 10, 545-555 (2010).

6. James, S. P. Some general results of a study of induced malaria in England. Trans. R. Soc. Trop. Med. Hyg. 24, 477-525 (1931).

7. Powell, R. D. \& McNamara, J. V. Infection with chloroquine-resistant Plasmodium falciparum in man: prepatent periods, incubation periods, and relationships between parasitemia and the onset of fever in nonimmune persons. Ann. NY Acad. Sci. 174 1027-1041 (1970).

8. Trager, W. $\&$ Jensen, J. B. Human malaria parasites in continuous culture. Science 193, 673-675 (1976).

9. Ifediba, T. \& Vanderberg, J. P. Complete in vitro maturation of Plasmodium falciparum gametocytes. Nature 294, 364-366 (1981).

10. Campbell, C. C., Collins, W. E., Nguyen-Dinh, P., Barber, A. \& Broderson, J. R. Plasmodium falciparum gametocytes from culture in vitro develop to sporozoites that are infectious to primates. Science 217, 1048-1050 (1982).

11. Chulay, J. D. et al. Malaria transmitted to humans by mosquitoes infected from cultured Plasmodium falciparum. Am. J. Trop. Med. Hyg. 35, 66-68 (1986).

12. Herrington, D. A. et al. Safety and immunogenicity in man of a synthetic peptide malaria vaccine against Plasmodium falciparum sporozoites. Nature 328 257-259 (1987)

13. Ballou, W. R. et al. Safety and efficacy of a recombinant DNA Plasmodium falciparum sporozoite vaccine. Lancet 1, 1277-1281 (1987).

14. Church, L. W. et al. Clinical manifestations of Plasmodium falciparum malaria experimentally induced by mosquito challenge. J. Infect. Dis. 175 , 915-920 (1997).

15. Moorthy, V. S. et al. Report of a consultation on the optimization of clinical challenge trials for evaluation of candidate blood stage malaria vaccines, 18-19 March 2009, Bethesda, MD, USA. Vaccine 27, 5719-5725 (2009)

16. Kester, K. E. et al. Efficacy of recombinant circumsporozoite protein vaccine regimens against experimental Plasmodium falciparum malaria. J. Infect. Dis. 183, 640-647 (2001).

17. Casares, S., Brumeanu, T. D. \& Richie, T. L. The RTS,S malaria vaccine. Vaccine 28, 4880-4894 (2010).

18. Kester, K. E. et al. Phase 2a trial of 0,1 , and 3 month and 0,7 , and 28 day immunization schedules of malaria vaccine RTS,S/ASO2 in malaria-naive adults at the Walter Reed Army Institute of Research. Vaccine 26, 2191-2202 (2008)

19. Bojang, K. A. et al. Efficacy of RTS,S/ASO2 malaria vaccine against Plasmodium falciparum infection in semi-immune adult men in The Gambia: a randomised trial. Lancet 358, 1927-1934 (2001).

20. Kester, K. E. et al. Randomized, double-blind phase 2 a trial of falciparum malaria vaccines RTS,S/ AS01B and RTS,S/AS02A in malaria-naive adults: safety, efficacy, and immunologic associates of protection. J. Infect. Dis. 200, 337-346 (2009)

21. Moorthy, V. S. et al. A randomised, double-blind, controlled vaccine efficacy trial of DNA/MVA ME-TRAP against malaria infection in Gambian adults. PLoS Med. 1, e33 (2004).

22. Dunachie, S. J. et al. A DNA prime-modified vaccinia virus ankara boost vaccine encoding thrombospondin-related adhesion protein but not circumsporozoite protein partially protects healthy malaria-naive adults against Plasmodium falciparum sporozoite challenge. Infect. Immun. $\mathbf{7 4 ,}$ 5933-5942 (2006). 
23. Shute, P. G. et al. A strain of Plasmodium vivax characterized by prolonged incubation: the effect of numbers of sporozoites on the length of the prepatent period. Trans. R. Soc. Trop. Med. Hyg. 70, 474-481 (1976).

24. Lawrence, G. et al. Effect of vaccination with 3 recombinant asexual-stage malaria antigens on initial growth rates of Plasmodium falciparum in non-immune volunteers. Vaccine 18, 1925-193 (2000).

25. Genton, B. et al. Safety and immunogenicity of a threecomponent blood-stage malaria vaccine (MSP1, MSP2 RESA) against Plasmodium falciparum in Papua New Guinean children. Vaccine 22, 30-41 (2003).

26. Epstein, J. E. et al. Safety and clinical outcome of experimental challenge of human volunteers with Plasmodium falciparum-infected mosquitoes: an update. J. Infect. Dis. 196, 145-154 (2007)

27. Verhage, D. F. et al. Clinical outcome of experimental human malaria induced by Plasmodium falciparuminfected mosquitoes. Neth. J. Med. 63, 52-58 (2005).

28. Rickman, L. S. et al. Plasmodium falciparum-infected Anopheles stephensi inconsistently transmit malaria to humans. Am. J. Trop. Med. Hyg. 43, 441-445 (1990).

29. Nieman, A. E. et al. Cardiac complication after experimental human malaria infection: a case report. Malar. J. 8, 277 (2009).

30. Hermsen, C. C. et al. Detection of Plasmodium falciparum malaria parasites in vivo by real-time quantitative PCR. Mol. Biochem. Parasitol. 118 , 247-251 (2001)

31. Felger, I., Genton, B., Smith, T., Tanner, M. \& Beck, H. P. Molecular monitoring in malaria vaccine trials. Trends Parasitol. 19, 60-63 (2003).

32. Hermsen, C. C. et al. Testing vaccines in human experimental malaria: statistical analysis of parasitemia measured by a quantitative real-time polymerase chain reaction. Am. J. Trop. Med. Hyg. 71, 196-201 (2004).

33. Dietz, K., Raddatz, G. \& Molineaux, L. Mathematical model of the first wave of Plasmodium falciparum asexual parasitemia in non-immune and vaccinated individuals. Am. J. Trop. Med. Hyg. 75, 46-55 (2006).

34. Bejon, P. et al. Calculation of liver-to-blood inocula, parasite growth rates, and preerythrocytic vaccine efficacy, from serial quantitative polymerase chain reaction studies of volunteers challenged with malaria sporozoites. J. Infect. Dis. 191, 619-626 (2005).

35. Spring, M. D. et al. Phase $1 / 2$ a study of the malaria vaccine candidate apical membrane antigen-1 (AMA-1) administered in adjuvant system AS01B or AS02A. PLOS ONE 4, e5254 (2009).

36. Silvie, O. et al. A role for apical membrane antigen during invasion of hepatocytes by Plasmodium falciparum sporozoites. J. Biol. Chem. 279, 9490-9496 (2004).

37. Cheng, Q. et al. Measurement of Plasmodium falciparum growth rates in vivo: a test of malaria vaccines. Am. J. Trop. Med. Hyg. 57, 495-500 (1997).

38. Sanderson, F. et al. Blood-stage challenge for malaria vaccine efficacy trials: a pilot study with discussion of safety and potential value. Am. J. Trop. Med. Hyg. 78 878-883 (2008)

39. Pombo, D. J. et al. Immunity to malaria after administration of ultra-low doses of red cells infected with Plasmodium falciparum. Lancet 360, 610-617 (2002).

40. Heppner, D. G. et al. Safety, immunogenicity, and efficacy of Plasmodium falciparum repeatless circumsporozoite protein vaccine encapsulated in liposomes. J. Infect. Dis. 174, 361-366 (1996).

41. Manson, P. Experimental proof of the mosquitomalaria theory. BMJ 2, 949-951 (1900).

42. Herrera, S. et al. Successful sporozoite challenge model in human volunteers with Plasmodium vivax strain derived from human donors. Am. J. Trop. Med. Hyg. 81, 740-746 (2009)

43. Roestenberg, M. et al. Protection against a malaria challenge by sporozoite inoculation. N. Engl. J. Med. 361, 468-477 (2009)

44. Takala, S. L. \& Plowe, C. V. Genetic diversity and malaria vaccine design, testing and efficacy: preventing and overcoming 'vaccine resistant malaria' Parasite Immunol. 31, 560-573 (2009).

45. Genton, B. et al. Randomized double-blind controlled Phase I/Ila trial to assess the efficacy of malaria vaccine PfCS102 to protect against challenge with $P$. falciparum. Vaccine $\mathbf{2 8}$ 6573-6580 (2010)

46. Trape, J. F. \& Zoulani, A. Malaria and urbanization in central Africa: the example of Brazzaville. Part II: Results of entomological surveys and epidemiological analysis. Trans. R. Soc. Trop. Med. Hyg. 81 (Suppl. 2), 10-18 (1987)

47. Rosenberg, R., Wirtz, R. A., Schneider, I. \& Burge, R. An estimation of the number of malaria sporozoites ejected by a feeding mosquito. Trans. $R$. Soc. Trop. Med. Hyg. 84, 209-212 (1990).

48. Jin, Y., Kebaier, C. \& Vanderberg, J. Direct microscopic quantification of dynamics of Plasmodium berghei sporozoite transmission from mosquitoes to mice. Infect. Immun. 75, 5532-5539 (2007)

49. Ponnudurai, T., Lensen, A. H., van Gemert, G. J., Bolmer, M. G. \& Meuwissen, J. H. Feeding behaviour and sporozoite ejection by infected Anopheles stephensi. Trans. R. Soc. Trop. Med. Hyg. 85, 175-180 (1991).

50. Beier, J. C. et al. Quantitation of malaria sporozoites transmitted in vitro during salivation by wild Afrotropical Anopheles. Med. Vet. Entomol. 5, 71-79 (1991)

51. Frischknecht, F. et al. Imaging movement of malaria parasites during transmission by Anopheles mosquitoes. Cell. Microbiol. 6, 687-694 (2004).

52. Jeffery, G. M., Young, M. D., Bergess, R. W. \& Eyles, D. E. Early activity in sporozoite-induced Plasmodium falciparum infections. Ann. Trop. Med. Parasitol. 53, 51-58 (1959).

53. James, S. P., Nicol, W. D. \& Shute, P. G. Note on a new procedure for malaria research. Trans. R. Soc. Trop. Med. Hyg. 21, 233-236 (1927).

54. Shute, P. G. A technique of the inoculation of known numbers of sporozoites as an aid to malaria research. Ann. Trop. Med. Parasitol. 31, 85-87 (1937).

55. Hoffman, S. L. et al. Development of a metabolically active, non-replicating sporozoite vaccine to prevent Plasmodium falciparum malaria. Hum. Vaccin. 6 , 97-106 (2010)

56. Lyke, K. E. et al. Plasmodium falciparum malaria challenge by the bite of aseptic Anopheles stephensi mosquitoes: results of a randomized infectivity trial. PLOS ONE 5, e 13490 (2010).

57. Grady, C. Ethics of vaccine research. Nature Immunol. 5, 465-468 (2004)

58. Visser, H. K. Experimental malaria in human volunteers: ethical aspects. Neth. J. Med. 63, 41-42 (2005)

59. Stevenson, M. M. \& Riley, E. M. Innate immunity to malaria. Nature Rev. Immunol. 4, 169-180 (2004).

60. Hoffman, S. L. et al. Protection of humans against malaria by immunization with radiation-attenuated Plasmodium falciparum sporozoites. J. Infect. Dis. 185, 1155-1164 (2002)

61. Herrington, D et al. Successful immunization of humans with irradiated malaria sporozoites: humoral and cellular responses of the protected individuals. Am. J. Trop. Med. Hyg. 45, 539-547 (1991).

62. Herrington, D. A. et al. Human studies with synthetic peptide sporozoite vaccine (NANP)3-TT and immunization with irradiated sporozoites. Bull. World Health Organ. 68 (Suppl.), 33-37 (1990).

63. Rieckmann, K. H. Human immunization with attenuated sporozoites. Bull. World Health Organ. 68 (Suppl). 13-16 (1990).

64. Clyde, D. F. Immunity to falciparum and vivax malaria induced by irradiated sporozoites: a review of the University of Maryland studies, 1971-1975. Bull. World Health Organ. 68 (Suppl.), 9-12 (1990).

65. Egan, J. E. et al. Humoral immune responses in volunteers immunized with irradiated Plasmodium falciparum sporozoites. Am. J. Trop. Med. Hyg. $\mathbf{4 9}$ 166-173 (1993)

66. Fries, L. F. et al. Safety, immunogenicity, and efficacy of a Plasmodium falciparum vaccine comprising a circumsporozoite protein repeat region peptide conjugated to Pseudomonas aeruginosa toxin A Infect. Immun. 60, 1834-1839 (1992).

67. Hoffman, S. L. et al. Safety, immunogenicity, and efficacy of a malaria sporozoite vaccine administered with monophosphoryl lipid A, cell wall skeleton of mycobacteria, and squalane as adjuvant. Am. J. Trop. Med. Hyg. 51, 603-612 (1994).

68. Gordon, D. M. et al. Safety, immunogenicity, and efficacy of a recombinantly produced Plasmodium falciparum circumsporozoite protein-hepatitis B surface antigen subunit vaccine. J. Infect. Dis. 171, 1576-1585 (1995).

69. Stoute, J. A. et al. A preliminary evaluation of a recombinant circumsporozoite protein vaccine against Plasmodium falciparum malaria. N. Engl. J. Med. 336, 86-91 (1997).

70. Kester, K. E. et al. A phase $\mathrm{I} / \mathrm{ll}$ la safety, immunogenicity, and efficacy bridging randomized study of a two-dose regimen of liquid and lyophilized formulations of the candidate malaria vaccine RTS,S/AS02A in malaria-naive adults. Vaccine $\mathbf{2 5}$, 5359-5366 (2007).

71. Walther, M. et al. Safety, immunogenicity, and efficacy of prime-boost immunization with recombinant poxvirus FP9 and modified vaccinia virus Ankara encoding the full-length Plasmodium falciparum circumsporozoite protein. Infect. Immun. 74, 2706-2716 (2006)

72. Walther, M. et al. Safety, immunogenicity and efficacy of a pre-erythrocytic malaria candidate vaccine, ICC-1132 formulated in Seppic ISA 720. Vaccine 23 , 857-864 (2005)

73. Dunachie, S. J. et al. A clinical trial of prime-boost immunisation with the candidate malaria vaccines RTS,S/AS02A and MVA-CS. Vaccine 24, 2850-2859 (2006).

74. Webster, D. P. et al. Enhanced T cell-mediated protection against malaria in human challenges by using the recombinant poxviruses FP9 and modified vaccinia virus Ankara. Proc. Natl Acad. Sci. USA 102 4836-4841 (2005).

75. McConkey, S. J. et al. Enhanced T-cell immunogenicity of plasmid DNA vaccines boosted by recombinant modified vaccinia virus Ankara in humans. Nature Med. 9, 729-735 (2003).

76. Cummings, J. F. et al. Recombinant liver stage antigen-1 (LSA-1) formulated with AS01 or ASO2 is safe, elicits high titer antibody and induces IFN- $\gamma /$ IL-2 $\mathrm{CD} 4^{+} \mathrm{T}$ cells but does not protect against experimental Plasmodium falciparum infection. Vaccine 28, 5135-5144 (2010).

77. Ockenhouse, C. F. et al. Phase I/lla safety, immunogenicity, and efficacy trial of NYVAC-Pf7, a pox-vectored, multiantigen, multistage vaccine candidate for Plasmodium falciparum malaria. J. Infect. Dis. 177, 1664-1673 (1998).

78. Thompson, F. M. et al. Evidence of blood stage efficacy with a virosomal malaria vaccine in a phase Ila clinical trial. PLOS ONE 3, e1493 (2008).

79. Patarroyo, M. E. et al. A synthetic vaccine protects humans against challenge with asexual blood stages of Plasmodium falciparum malaria. Nature 332, 158-161 (1988)

80. Wang, R. et al. Boosting of DNA vaccine-elicited gamma interferon responses in humans by exposure to malaria parasites. Infect. Immun. 73, 2863-2872 (2005).

81. Heppner, D. G. Jr et al. Towards an RTS,S-based, multi-stage, multi-antigen vaccine against falciparum malaria: progress at the Walter Reed Army Institute of Research. Vaccine 23, 2243-2250 (2005).

\section{Acknowledgements}

The views expressed in this manuscript are those of the authors and should not be taken to represent the views or stated policy of the World Health Organization.

\section{Competing interests statement}

The authors declare no competing financial interests.

\section{FURTHER INFORMATION}

ClinicalTrials.gov: http://clinicaltrials.gov

ICH: http://www.ich.org

Malaria Vaccine Technology Roadmap Process:

http://www.malariavaccine.org/files/Malaria Vaccine TRM Exec Summary Final 000.pdf

The Malaria Product Pipeline: Planning for the Future: www.policycures.org/downloads/The malaria product pipeline planning for the future.pdf

WHO Guidelines for the Treatment of Malaria: http://www. who.int/malaria/publications/atoz/9789,241,547,925/en/ index.html

WHO Malaria Vaccine Rainbow Tables: www.who.int/vaccine research/links/Rainbow/en/index. html WHO World Malaria Report 2009:

http://www.who.int/malaria

ALL LINKS ARE ACTIVE IN THE ONLINE PDF 